\title{
Pulmonary Fibrosis in Sarcoidosis
}

Joel Francesqui, MD, MSc'1, Sanjay Kalra, MD², and Eva M Carmona, MD, PhD²

${ }^{1}$ Servei de Pneumologia, Interstitial Lung Disease Program, Respiratory Institute, Hospital Clínic, Barcelona, Spain; ${ }^{2}$ Division of Pulmonary and Critical Care Medicine, Mayo Clinic, Rochester, Minnesota, USA

\section{ABSTRACT}

Sarcoidosis is a systemic granulomatous disease with an intrathoracic involvement in about $90 \%$ of the cases. Self-limited disease is the most common presentation; however, $10-20 \%$ of these patients may progress to fibrotic forms, so-called advanced pulmonary sarcoidosis or end-stage sarcoidosis. Recognition of this phenotype is important as it carries a poor prognosis and a limited response to treatment.

Clinical manifestations of fibrotic sarcoidosis include common symptoms like dyspnea and chronic cough. Radiologically, typical fibrotic patterns include bronchial distortion, honeycomb and diffuse linear fibrosis. Unlike non-fibrotic sarcoidosis, aspergillus, bronchiectasis-related bacterial infections, venous thromboembolic disease and pulmonary hypertension are complications most commonly associated with this fibrotic stage.

Resistance to usual treatments is a known feature, but recent evidence shows that antifibrotic agents such as Nintedanib may have a role to slow pulmonary function deterioration. In the absence of active disease, referral for lung transplant evaluation is recommended. (BRN Rev. 2021;7(2):125-36)

Corresponding author: Eva M Carmona, carmona.eva@mayo.edu

Key words: Advanced pulmonary sarcoidosis. Antifibrotic agents. Pulmonary fibrosis. Sarcoidosis. 


\section{INTRODUCTION}

Sarcoidosis is a systemic granulomatous disease of unknown cause that predominantly affects young and middle-aged adults. The prevalence varies widely depending on the region of the world. For example, in Sweden the reported prevalence is $140-160$ per $100,000^{1}$ while in Asian countries, such as South Korea, Taiwan and Japan, it is only 1-5 per $100,000^{2}$. Scandinavian countries, and in particular Sweden, report higher incidence rates $(11.5 \text { per } 100,000)^{1}$, although this is similar to the white population in the United States, with an incidence of 10 per 100,000; rates in African-Americans are, however, as much as 3-4 times higher ${ }^{3}$.

While the etiology of sarcoidosis remains unknown, exposure to different environmental/ infectious factors in the genetically predisposed individual are likely to associate with different individual susceptibility, patterns of disease and risk of progression ${ }^{4-6}$. This was specifically evaluated in the A Case Control Etiologic Study of Sarcoidosis (ACCESS) study study $^{7}$, a case-control study of about 700 sarcoidosis patients in which the association of sarcoidosis with different occupational and environmental factors was extensively evaluated. Positive associations were found with insecticides, microbial bioaerosols and agriculture, although the study did not identify a single predominant cause of sarcoidosis.

\section{PULMONARY DISEASE}

Sarcoidosis can affect almost any organ in the body; however, intrathoracic involvement is seen in more than $90 \%$ of the patients ${ }^{8}$. The presentation may vary from a radiographic abnormality detected in an asymptomatic individual to a progressive pulmonary disorder causing lung fibrosis and respiratory failure ${ }^{9}$. The most common presentation is bilateral hilar and mediastinal adenopathy with or without pulmonary parenchymal opacities. The majority of patients have a self-limiting disease but between $10-20 \%$ of pulmonary sarcoidosis patients may progress and develop significantfibrosis ${ }^{10,11}$. It is important to identify and understand this phenotype because of its poorer prognosis, complication risks and resistance to usual treatments.

When referring to pulmonary fibrosis in the context of sarcoidosis, one finds different terminology including "Stage IV sarcoidosis", "endstage sarcoidosis", "chronic sarcoidosis" or "advanced pulmonary sarcoidosis (APS)"7,11-13. All of these define pulmonary fibrosis as the cornerstone of disease, and they are used interchangeably in this text.

\section{PATHOLOGIC MECHANISMS}

The specific reason why granulomatous inflammation progresses to fibrosis in a subgroup of patients is not fully understood. It has been proposed that fibrosis might be triggered early in the disease by profibrotic inflammatory events or by an inherent predisposition to fibrosis. In the same way, it has been hypothesized that fibrosis might develop as a wound-healing response to uncontrolled chronic inflammation ${ }^{13,14}$. Various fibrotic pathways have been proposed for the development of sarcoidosis-associated fibrosis, such as the implication of transforming growth factor-beta (TGF- $\beta$ ), cytokines such as 
interleukin (IL)-4, IL13, IL1- $\beta$, IL-17A and interferon-gamma (IFN- $\gamma$ ), the transition from a T-helper-1 (Th1) to T-helper-2 (Th2) signature and the up-regulation of profibrotic genes ${ }^{10,13}$. Alveolar macrophages are also considered important in the development of such fibrosis. In a model of skin foreign body granuloma for instance, newer evidence suggests that vascular endothelial growth factor-A (VEGF-A) secreted by macrophages induces angiogenesis facilitating fibroblasts recruitment to the granuloma. Additionally, macrophages secrete CXCL13 which indeed attracts fibrosing promoting B-cells ${ }^{15}$. Along with these interesting observations, our group has recently found that B-cells activated by infectious antigens induced fibroblast migration and activation via IL-6 and VEGF-A pathways ${ }^{16}$. Mammalian target of rapamycin (mTOR) activation is also likely to play a major role in the formation and maintenance of granulomas through its likely contribution to cytokine regulation ${ }^{17}$. Our own data in B-cells suggest that inhibition of mTOR leads to a decrease in IL-6 and VEGF-A resulting in a decrease in fibroblasts migration and activation. These are in vitro studies and while they do provide some insights into the basic mechanisms of fibrosis, further investigations are necessary in animals models of disease.

\section{CLINICAL MANIFESTATIONS}

The most common clinical manifestations of patients with APS are dyspnea and chronic cough, similar to non-fibrotic sarcoidosis. Other clinical symptoms of end-stage sarcoidosis are chronic purulent sputum production and hemoptysis and they are suggestive of bronchiectasis or superimposed infection ${ }^{12,18}$. On exam, crackles are found in about $30 \%$ of patients.
Notably, patients with end-stage sarcoidosis might also have wheezing, mainly attributed to airway-centered fibrosis and bronchial hyperreactivity. Other rare clinical features are the presence of digital clubbing and skin manifestations.

APS is usually recognized within an average time of six years from the time of diagnosis. The mean age of patients is nearly in the $5^{\text {th }}$ decade $^{18}$.

\section{RADIOGRAPHIC FEATURES}

Thoracic images of patients with APS usually reveal classic fibrotic findings. Volume loss and architectural distortion from extensive fibrosis are easily recognized on chest radiographs (CXR). However, poor sensitivity and poor inter-observer agreement on radiographic staging make the use of high-resolution computer tomography (HRCT) scans preferable $\mathrm{p}^{19,20}$.

HRCT scans may be useful in distinguishing active inflammation from irreversible fibrosis in patients with CXR stage 2 or $3^{21}$.

Fibrotic changes predominantly involve middle and upper lung zones. Bilateral lower lobe subpleural honeycombing such as idiopathic pulmonary fibrosis (IPF) can occur, which is uncommon however ${ }^{22}$. Abehsera et al. ${ }^{23}$ described the main chest CT findings in 80 patients with stage IV sarcoidosis ${ }^{24}$. Bronchial distortion, fissure displacement, agglomerated nodular fibrosis suggesting masses, linear scarring, traction bronchiectasis, cysts and honeycombing were among the most common findings. Unlike the usual and well-established 


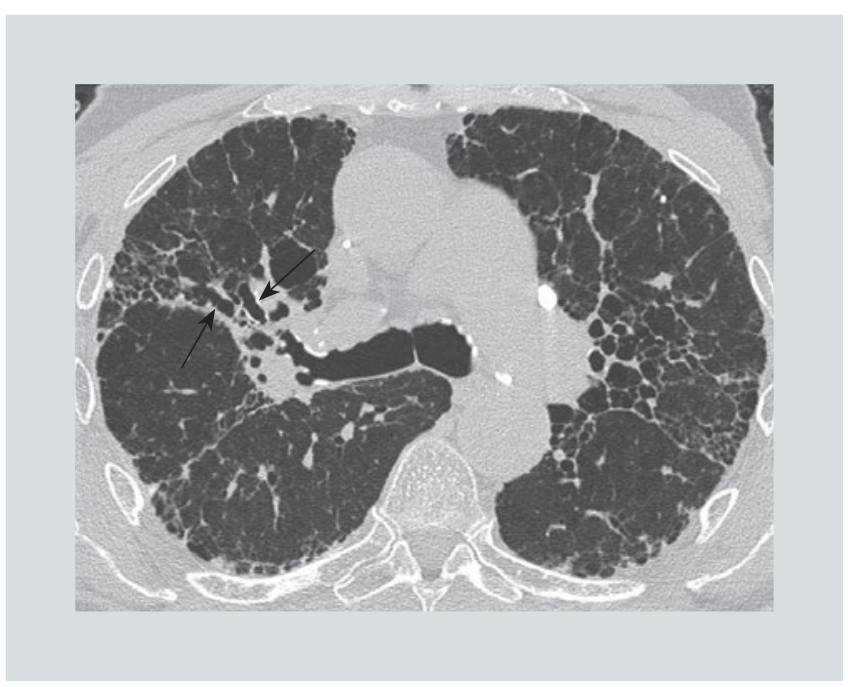

Figure 1. Bronchial distortion pattern with central traction bronchiectasis. White arrows show central traction bronchiectasis.

findings of IPF, they described three specific CT patterns in APS fibrotic disease: the most common was the bronchial distortion pattern, which predominantly presented with deformation and displacement of the central airways (Fig. 1); the honeycomb pattern, predominantly peripheral and often in the upper zones (Fig. 2) and the diffuse linear fibrosis pattern characterized by septal reticulation with distorted linear opacities of irregular thickness, mainly in the periphery (Fig. 3) ${ }^{23}$.

Beyond the CT findings of the late phase of APS, it is important to recognize early radiologic features which might alert clinicians to the increased risk of progressing to a fibrotic phenotype. Akira et al. ${ }^{25}$ specifically found that conglomeration of nodules into large nodules or masses evolved into bronchial distortion and ground glass opacities and consolidations often evolved into honeycombing ${ }^{25}$. In sarcoidosis, however, honeycombing is often used to describe large and more central cysts, described as fibrocystic sarcoidosis ${ }^{13}$.

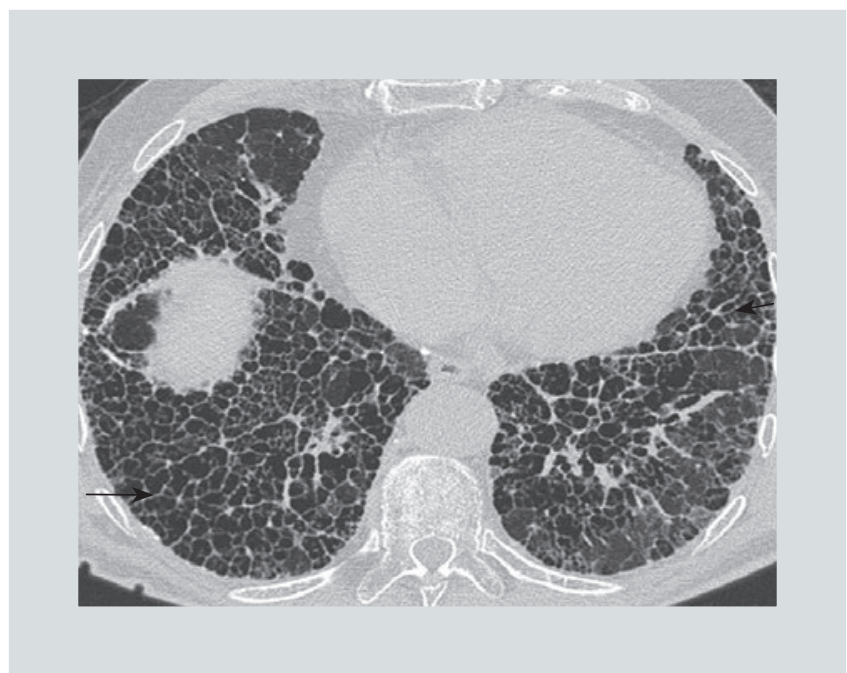

Figure 2. Diffuse honeycomb pattern. Arrows show large cysts distributed in both upper and lower lobes.

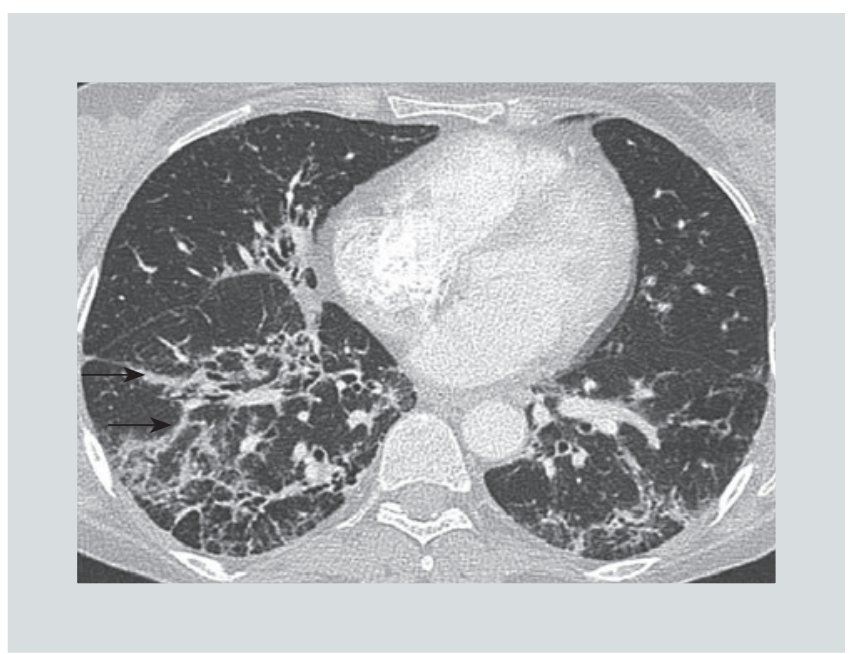

Figure 3. Diffuse linear fibrosis pattern. Arrows show distorted linear opacities of irregular thickness.

These features are consistent with the loss of terminal airway segments and suggest that the pathophysiology extends beyond alveolar interstitial damage ${ }^{20}$. However, whether these specific parenchymal features can predict the development of fibrosis is not yet known.

In addition to CXR and HRCT imaging, over the last decade, there has also been an increasing interest in using 18-2-fluoro-2-deoxy-D-glucose 


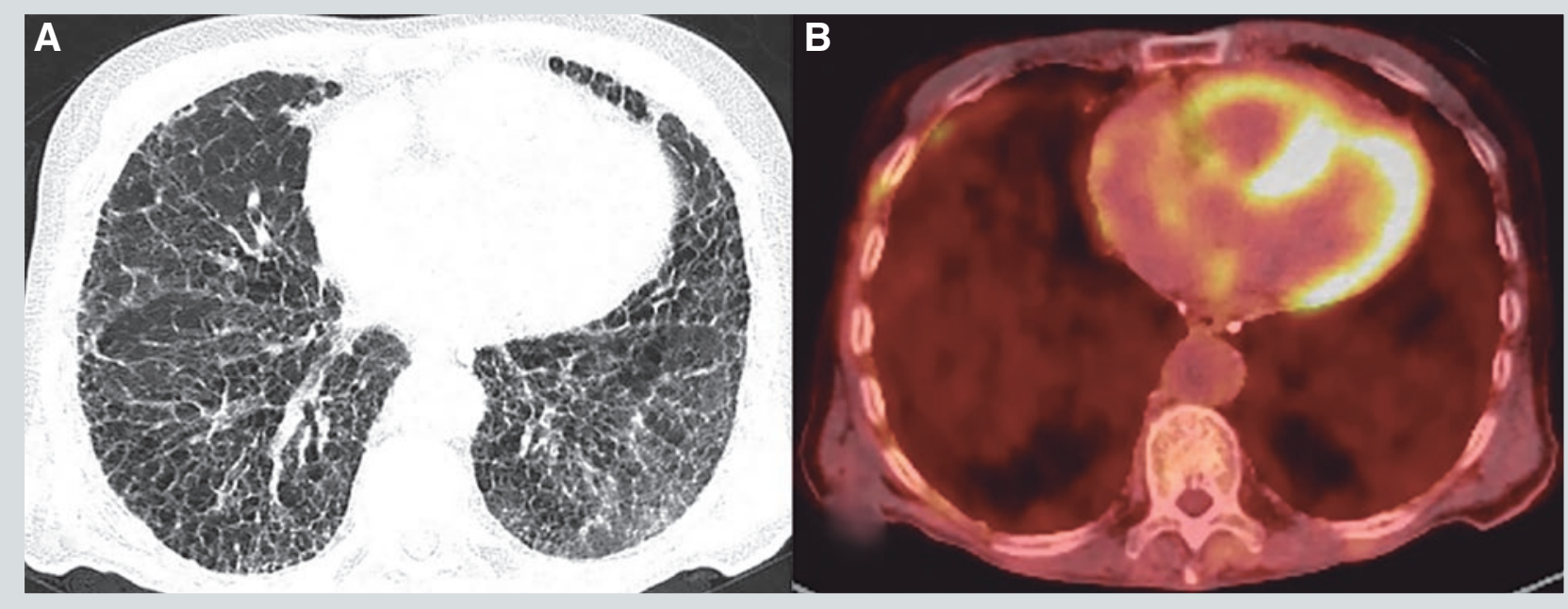

FIGURE 4. (A) HRCT image of a fibrotic pattern with diffuse honeycomb in the lower lobes. (B) Corresponding FDG-PET image with no FDG uptake within the areas of lung fibrosis. Physiologic FDG uptake is observed in the heart.

FDG-PET: fluorodeoxyglucose-positron emission tomography; HRCT: high-resolution computer tomography.

positron emission tomography (FDG-PET) to evaluate disease activity.

Inflammatory cell activity in sarcoid granulomas results in hypermetabolic lesions due to the increase in FDG uptake. Although hypermetabolic lesions are nonspecific of sarcoidosis and may resemble those seen in lymphoproliferative diseases or metastatic neoplasms ${ }^{22}$ FDG-PET can facilitate the identification of sites amenable to biopsy at the time of diagnosis. It also permits identification of extrathoracic involvement and evaluation of treatment response $\mathrm{e}^{22,26}$.

In patients with a fibrotic phenotype, the use of FDG-PET to assess disease activity is however contradictory. In a retrospective series of 95 patients, Mostard et al. ${ }^{27}$ found pulmonary positive FDG-PET scans in 56 patients (59\%), 26 of them with fibrotic features. Of those with fibrotic features, $22(85 \%)$ had positive pulmonary FDG-PET findings. These results differ from a larger retrospective study ${ }^{28}$, in which fibrotic sarcoidosis had a lower rate of FDG-PET positivity (Fig. 4). Only 2 of 22 patients with stage IV disease had increased pulmonary FDG-PET uptake.

\section{PULMONARY FUNCTION}

In APS patients, pulmonary function tests (PFT) reveal a restrictive pattern with decreased forced vital capacity (FVC) in almost two-thirds of the patients although a minority have an obstructive pattern. Reduced diffusing capacity of carbon monoxide (DLCO) was common in almost all patients ${ }^{18,19}$. The degree of pulmonary function decline overtime is not clearly established for fibrotic pulmonary sarcoidosis. Moreover, the correlation between $\mathrm{CT}$ scan progression and worsening PFT remains unclear as only moderate agreement has been found between progression on CT scans and PFT trends ${ }^{29,30}$. 


\section{HISTOPATHOLOGY}

Fibrosis in sarcoidosis appears to start in and extend from areas of granulomas and active inflammation. As fibrosis progresses it is not unusual to observe only a few or even no remaining granulomas ${ }^{10}$. Fibrosis along broncho-vascular tracks results in bronchial distortion and large fibrocystic areas as well as interlobular septal fibrosis and linear scarring ${ }^{31}$.

In general, the histological features of fibrotic sarcoidosis are distinctly different from usual interstitial pneumonia (UIP). While fibroblastic foci may be present in fibrotic sarcoidosis, fibroblast foci in transition zones and other features of UIP, such as bronchiolar honeycombing, are rarely seen. In some series of explanted lungs, however, findings indistinguishable from the UIP pattern, overlapping features of sarcoidosis and UIP, or UIP alone have been found ${ }^{32-34}$ but these may represent the very late stages of burnt-out fibrosis. It is, therefore, reasonable to include a UIP pattern as a potential manifestation of sarcoidosis, just as it is in other fibrotic lung diseases such as hypersensitivity pneumonitis and interstitial lung diseases associated with connective tissue disease. Further studies are needed to improve our understanding of tissue fibrosis and in particular the correlation of histopathological features with imaging studies ${ }^{10}$.

\section{COMPLICATIONS}

APS may present with several complications. Most of them are secondary to the fibrotic process, such as parenchymal restriction, bronchial stenosis, bronchiectasis, pneumothorax and pleural thickening. Aspergillus infections, both invasive and aspergillomas in cavities, bronchiectasis-related bacterial infections, venous thromboembolic disease and sarcoidosis associated pulmonary hypertension are also commonly seen in advanced disease ${ }^{20}$.

Of these, our review will focus on sarcoidosis-associated pulmonary hypertension and aspergillus infection, as they have the greatest impact on the mortality and prognosis of these patients.

\section{SARCOIDOSIS-ASSOCIATED PULMONARY HYPERTENSION (SAPH)}

The incidence of $\mathrm{SAPH}$ is about $5 \%$, although with the newly updated definition of pulmonary hypertension (mean pulmonary artery pressure $[\mathrm{mPAP}]>20 \mathrm{mmHg})^{35}$ the actual incidence would even be higher. SAPH is allocated into group $5 \mathrm{~s}$ of the updated pulmonary hypertension classification, which means the precise origin is unclear and multifactorial.

Several mechanisms are therefore implicated in the development of SAPH. For instance, SAPH can be present in the context of pulmonary venous hypertension from subjacent cardiac sarcoidosis or from heart disease unrelated to sarcoidosis. As sarcoidosis patients are at a relatively higher risk of pulmonary embolism, SAPH can also be seen in the setting of chronic thromboembolic disease. In some patients, the pulmonary vasculature is directly affected by either the granulomatous inflammatory process itself or by compression from enlarged mediastinal lymph nodes. Finally, and the most accepted hypothesis, is by pulmonary fibrosis causing 
distortion of the pulmonary vasculature as well as through accompanying hypoxia like other fibrotic interstitial lung diseases. This mechanism belongs to group 3 of the classification and is a pre-capillary form of pulmonary hypertension ${ }^{13,36}$.

The main clinical manifestations of SAPH are exertional dyspnea, chest pain, and syncope. It is recommended to assess for pulmonary hypertension in patients with dyspnea out of proportion to underlying lung disease, or when a reduction in the DLCO is disproportionate to reductions in other pulmonary function indices.

The echocardiogram is the initial test for evaluation, although right heart catheterization is the gold standard method of diagnosing this condition ${ }^{20,36}$.

SAPH creates a seven-fold increase in mortality in sarcoidosis, compared with sarcoidosis without this condition. Pulmonary hypertension and disease treatment studies still reflect conflicting data about efficacy.

Corticosteroids and other immunosuppressive therapies are used frequently; however, the indications for these in SAPH are unclear as there is no obvious demonstrable therapeutic hemodynamic benefit from their use. Systemic pulmonary vasodilators may lead to worsening hypoxemia because of the inhibition of hypoxic pulmonary vasoconstriction and worsening of ventilation and perfusion $(\mathrm{V} / \mathrm{Q})$ mismatch and shunting. Also, there is no clear convincing data with respect to endothelin receptor antagonists (bosentan, macitentan), phosphodiesterase 5 inhibitors (sildelnafil or tadalafil) or soluble guanylate cyclase stimulators (riociguat) ${ }^{36-40}$. Parenteral prostacyclin (intravenous epoprostenol or subcutaneous Treprostinil) however, showed a significant long-term clinical and hemodynamic improvement as monotherapy or in combination with other pulmonary hypertension therapies, without worsening V/Q mismatch, in a retrospective analysis of severe $\mathrm{SAPH}$ patients ${ }^{41}$. Combination medical therapy in an experienced center may be a valid option, despite only small and retrospective series supporting it.

Other options, like balloon pulmonary angioplasty (BPA) and stenting of pulmonary vasculature, to treat extrinsic compression due to mediastinal fibrosis, might be useful in selected patients ${ }^{36}$.

Lung transplantation is a reasonable therapeutic consideration for end-stage sarcoidosis and SAPH in the absence of contraindications due to severe extra-pulmonary sarcoidosis or comorbidities. SAPH with severe lung disease and FVC less than $50 \%$ responds poorly to drugs approved for pulmonary hypertension and should be considered for referral for lung transplantation ${ }^{42}$.

\section{ASPERGILLUS INFECTION}

Chronic pulmonary aspergillosis (CPA) complicates fibrocystic sarcoidosis in about $2 \%$ of the cases. The most common presentation is with intracavitary aspergillomas. These are mostly found in the upper lobes of sarcoidosis patients as that is where fibrocystic disease is most common. They often remain clinically stable, although hemoptysis, sometimes lifethreatening, is an important complication. 
Invasive or semi-invasive forms are not usually seen although sometimes a progressive aspergilloma can be difficult to distinguish from chronic necrotizing aspergillosis ${ }^{43,44}$.

These conditions may be unrecognized and increase the risk for poor outcomes, as they may be complicated by life-threatening hemoptysis, wasting syndrome, and/or progressive respiratory insufficiency. They also affect considerations for lung transplantation ${ }^{20}$.

Preexisting cavities are the most common risk factor for developing aspergillomas in sarcoidosis, just as in other chronic pulmonary diseases. It is also important to recognize the presence of contributory comorbidities commonly associated such as diabetes mellitus, alcoholism, neoplasms, and use of immunosuppressants and systemic corticosteroid ther$\operatorname{apy}^{43,45}$.

Identification of simple aspergillomas can often be made on imaging. Symptoms like weight loss, chronic productive cough, hemoptysis of variable severity, fatigue, fever, night sweats and/or shortness of breath may indicate complication or progression. The diagnosis is supported by the recovery of fungus from lung lavage or biopsy specimens or by repeatedly positive sputum cultures. Testing for IgG Aspergillus antibodies in serum is frequently positive as well as the presence (1,3)-beta-D-glucan. However, standardization of the latter is not well established for this indication. The erythrocyte sedimentation rate and C-reactive protein are often quite elevated ${ }^{46}$.

Treatment recommendations for pulmonary aspergillosis are derived from case reports and expert opinion statements ${ }^{47,48}$. There is no consensus on whether and when to treat patients with non-complicated aspergilloma. Observation may be a valid option in asymptomatic patients. The use of oral itraconazole and, more recently, voriconazole has been reported, resulting in some improvement in up to half to two-thirds of cases, with complete resolution of aspergilloma only in occasional patients $^{49}$.

In case of life-threatening bleeding, bronchial artery embolization is indicated. Surgical resection may be required in refractory hemoptysis, although the underlying lung impairment may be too advanced to allow extensive resection. Video-assisted thoracic surgery might be an alternative to thoracotomy for simple aspergilloma. Poor lung function and progressive aspergillosis are associated with postoperative hemorrhage, bronchopleural fistulas, pleural infection, and prolonged respiratory insufficiency ${ }^{46,47}$.

For progressive invasive aspergillosis, the use of systemic antifungal therapy can help control the disease, although a cure is not often achieved. The duration of treatment remains to be determined but it is generally extended to a minimum of six months ${ }^{47}$.

Adjuvant antifungal therapy, before and after surgery, may have a role in the outcome of surgical patients; however, there are no prospective studies available $\mathrm{e}^{50,51}$.

CT-guided intracavitary instillation of amphotericin or other antifungals are options in inoperable situations, although the needed large controlled studies are generally not feasible ${ }^{52}$. 
TABLE 1. Main immunosuppressive drugs used for pulmonary sarcoidosis.

\begin{tabular}{|l|l|l|}
\hline Drug & Usual dose & Side effects \\
\hline Glucocorticoids & $20-40 \mathrm{mg} /$ day & Hyperglycemia, weight increase, osteoporosis, hypertension, muscular atrophy. \\
\hline Methotrexate & $5-15 \mathrm{mg} /$ week & Teratogenicity, hepatitis, cytopenia, stomatitis, nausea, kidney toxicity, paresthesia. \\
\hline Azathioprine & $50-200 \mathrm{mg} / \mathrm{day}$ & Nausea, diarrhea, hepatitis, infections, cytopenia. \\
\hline Leflunomide & $10-20 \mathrm{mg} / \mathrm{day}$ & $\begin{array}{l}\text { Teratogenicity, cutaneous eruption, diarrhea, hepatitis, } \\
\text { alopecia, hypertension, neuropathy. }\end{array}$ \\
\hline Mycophenolate mofetil & $500-3000 \mathrm{mg} /$ day & Digestive symptoms, neutropenia, infections. \\
\hline Hydroxychloroquine & $200 \mathrm{mg} / \mathrm{day}$ & Retinal toxicity. \\
\hline Infliximab & $3-5 \mathrm{mg} / \mathrm{kg}$ week $0-2-6$ & Infection, development of drug antibodies. \\
\hline and $4-8$ weeks thereafter & & Similar to Infliximab. \\
\hline
\end{tabular}

\section{TREATMENT OF SARCOIDOSIS}

Pharmacological therapy for pulmonary sarcoidosis can be divided into three main lines. Glucocorticoids as the first-line option, immunosuppressants as the second line, and biologics as the third line ${ }^{53}$. In table 1 , we summarize the main currently used drugs, their doses and side effects.

The most important indications for systemic treatment in sarcoidosis are the risk of severe dysfunction of major organs or unacceptably impaired quality of life. Currently approved drugs suppress the granulomatous process, improve clinical and radiological parameters and preserve organ function ${ }^{54}$.

Delphi consensus recommendations settled oral glucocorticoids as first-line therapy ${ }^{55}$. Oral methotrexate was favored as initial non-biologic therapy in cases of severe disease, inadequate response to steroid therapy, the expectation of prolonged and/or high-dose steroid therapy and the occurrence of steroid toxicity. As a third line, the panel achieved consensus on some of the possible indications for adding a biologic agent (toxicity, insufficient response, and severe or progressive disease), with infliximab as the preferred option while maintaining a low dose of methotrexate to reduce the risk of developing autoantibodies.

The presence of fibrosis alone, without evidence of inflammatory infiltrates suggesting active disease, is not an indication for systemic treatment per $\mathrm{se}^{53}$. However, fibrosis may coexist with active inflammation, and, in these cases, immunosuppression may improve clinical status. Although previously described treatments are not known to prevent ongoing fibrosis, a reduction in superimposed inflammation may be clinically meaningful ${ }^{20}$.

The extension of pulmonary fibrosis is associated with higher mortality; early identification of these patients is important to prevent disease progression ${ }^{53}$.

In these settings, Walsh et al. ${ }^{56}$ developed an algorithm that integrates the composite physiologic index, the main pulmonary artery 
diameter to ascending aorta diameter ratio, and the extent of fibrosis on CT scan. This staging system predicted mortality (hazard ratio [HR] 5.89, confidence interval [CI] 2.68$10.08, \mathrm{p}<0.0001)$ better than any of the individual variables.

FDG-PET CT scan may also be a tool to help identify active inflammation within fibrotic disease and select patients who could still potentially respond to anti-inflammatory ther$\mathrm{apy}^{22}$. FDG-PET appears especially helpful in those persistently symptomatic patients without serological signs of inflammatory activity and in patients with radiologic signs of fibrosis ${ }^{57,58}$.

For advanced disease, even 'fourth-line' treatment options could be considered. Such agents include anti-CD20 antibody, anti-Janus Kinase (anti-JAK), IL-6 receptor monoclonal antibody, mTOR inhibitors, repository corticotropin, and antifibrotic agents ${ }^{11}$. The last may be the primary option when pulmonary fibrosis is established, as antifibrotic agents may help in preventing clinical deterioration.

Nintedanib is a tyrosine-kinase inhibitor that has been shown to reduce the rate of progression of fibrosis in patients with IPF and systemic sclerosis associated-interstitial lung disease (SSc-ILD) ${ }^{59,60}$. Recently, the prospective, randomized, placebo-controlled Efficacy and Safety of Nintedanib in Patients With Progressive Fibrosing Interstitial Lung Disease (INBUILD) trial evaluated the effect of nintedanib in patients with fibrosing ILDs other than IPF and showed slowing of ILD progression as measured by the rate of decline in FVC (mL/year), compared with placebo, in the overall population (difference of $107.0 \mathrm{ml}$ per year, 95\% CI, 65.4 to 148.5; $\mathrm{p}<0.001$ ), with adverse events that were like those observed in patients with IPF and SSc-ILD ${ }^{61}$.

A later subgroup analysis of the INBUILD trial, which included 12 sarcoidosis patients, suggested that nintedanib also reduced the rate of ILD progression, again measured by FVC decline, in patients who have chronic fibrosing disease and progressive phenotype, irrespective of the underlying ILD diagnosis $^{62}$. Other antifibrotic agents like pirfenidone, are still undergoing clinical trials and until data from larger studies is available, a multidisciplinary discussion to determine when these drugs should be utilized is advised $^{11}$.

Finally, in the absence of active disease, referral for lung transplant evaluation should be considered ${ }^{53}$. Lung transplant can be successfully performed in patients with sarcoidosis-induced pulmonary fibrosis, and post-transplant survival is similar to other fibrotic ILDs. However, appropriate timing of referral, comprehensive assessment of potential candidates for lung transplant, placement of patients on the lung transplant waiting list, choosing between bilateral versus single lung transplant, and optimal pre and post-transplant management are key to successful results for patients with sarcoidosis $^{63}$. Nevertheless, the number of patients with pulmonary sarcoidosis that are transplanted is low. Taimeh et al. ${ }^{64}$ described a cohort of United States of America (USA) lung-only first-time transplants between 1987 and 2012. Only 695 (3\%) from a total of 20,896 lung transplants were performed in pulmonary sarcoidosis patients. 
Recurrence of sarcoid granulomas in transplanted lungs is seen in approximately 35\% of the cases ${ }^{63,65}$; however, a recent retrospective European cohort of lung transplantation in sarcoidosis found $14 \%$ of post-transplant recurrence in lung allografts ${ }^{66}$.

Most recurrences are detected by post-transplant surveillance bronchoscopies in the first 6-12 months with no affection on the allograft function or overall survival ${ }^{67}$.

\section{PERSPECTIVES}

Pulmonary fibrosis in sarcoidosis is a condition with increased mortality which must be exhaustively investigated in patients with a progressive phenotype. Once fibrosis is established, it is not currently reversible, and it presents a challenging scenario for clinicians. APS can be complicated with other conditions like pulmonary hypertension and fungal infections that require specific algorithms of treatment, increasing the risks and complexities of management.

Antifibrotic agents may play an important role in reducing the fibrosis progression and may permit to "gain time" for those that may be candidates for lung transplantation, but further studies are necessary.

Understanding the pathophysiological mechanisms of fibrotic sarcoidosis and developing better predictor models of progression based on risk factors, histological-radiological correlation with pulmonary function and antifibrotic treatment would help to better care for these patients and improve the current therapeutic options.

\section{DISCLOSURES}

Dr. Francesqui and Dr. Kalra have nothing to disclose. Dr. Carmona received NIH grants R03 HL144427 and R01HL 62150-26.

\section{REFERENCES}

1. Arkema EV, Grunewald J, Kullberg S, Eklund A, Askling J. Sarcoidosis incidence and prevalence: a nationwide register-based assessment in Sweden. Eur Respir J. 2016;48:1690-9.

2. Arkema EV, Cozier YC. Sarcoidosis epidemiology: recent estimates of incidence, prevalence and risk factors. Curr Opin Pulm Med. 2020;26(5):527-534.

3. Carmona EM, Kalra S, Ryu JH. Pulmonary Sarcoidosis: Diagnosis and Treatment. Mayo Clin Proc. 2016;91:946-54.

4. Fernández Fabrellas E. Epidemiología de la sarcoidosis [Epidemiology of sarcoidosis]. Arch Bronconeumol. 2007;43:92-100.

5. Rabin DL, Thompson B, Brown KM et al. Sarcoidosis: social predictors of severity at presentation. Eur Respir J. 2004;24:601-8.

6. Schürmann M, Reichel P, Müller-Myhsok B, Schlaak M, Müller-Quernheim J, Schwinger E. Results from a genome-wide search for predisposing genes in sarcoidosis. Am J Respir Crit Care Med. 2001;164:840-6.

7. Newman LS, Rose CS, Bresnitz EA, et al. A case control etiologic study of sarcoidosis: environmental and occupational risk factors. Am J Respir Crit Care Med. 2004;170:1324-30.

8. Statement on sarcoidosis. Joint Statement of the American Thoracic Society (ATS), the European Respiratory Society (ERS) and the World Association of Sarcoidosis and Other Granulomatous Disorders (WASOG) adopted by the ATS Board of Directors and by the ERS Executive Committee, February 1999. Am J Respir Crit Care Med. 1999;160:736-55.

9. Judson MA. The Clinical Features of Sarcoidosis: A Comprehensive Review. Clin Rev Allergy Immunol. 2015;49:63-78.

10. Bonham CA, Strek ME, Patterson KC. From granuloma to fibrosis: sarcoidosis associated pulmonary fibrosis. Curr Opin Pulm Med. 2016;22:484-91.

11. Patel DC, Valeyre D. Advanced pulmonary sarcoidosis. Curr Opin Pulm Med. 2020;26:574-81.

12. Teirstein AT, Morgenthau AS. "End-stage" pulmonary fibrosis in sarcoidosis. Mt Sinai J Med. 2009;76:30-6.

13. Judson MA. Strategies for identifying pulmonary sarcoidosis patients at risk for severe or chronic disease. Expert Rev Respir Med. 2017;11:111-8.

14. Patterson KC, Hogarth K, Husain AN, Sperling AI, Niewold TB. The clinical and immunologic features of pulmonary fibrosis in sarcoidosis. Transl Res. 2012;160:321-31.

15. Pagán AJ, Ramakrishnan L. The Formation and Function of Granulomas Annu Rev Immunol. 2018;36:639-65.

16. Ali MF, Egan AM, Shaughnessy GF et al. Antifibrotics Modify B-Cell-induced Fibroblast Migration and Activation in Patients with Idiopathic Pulmonary Fibrosis. Am J Respir Cell Mol Biol. 2021;64:722-33.

17. Linke M, Pham HT, Katholnig K et al. Chronic signaling via the metabolic checkpoint kinase mTORC1 induces macrophage granuloma formation and marks sarcoidosis progression. Nat Immunol. 2017;18:293-302.

18. Nardi A, Brillet PY, Letoumelin P et al. Stage IV sarcoidosis: comparison of survival with the general population and causes of death. Eur Respir J. 2011;38:1368-73

19. Valeyre D, Nunes H, Bernaudin JF. Advanced pulmonary sarcoidosis. Curr Opin Pulm Med. 2014;20:488-95.

20. Patterson KC, Strek ME. Pulmonary fibrosis in sarcoidosis. Clinical features and outcomes. Ann Am Thorac Soc. 2013;10:362-70. 
21. Criado E, Sánchez M, Ramírez J et al. Pulmonary sarcoidosis: typical and atypical manifestations at high-resolution CT with pathologic correlation. Radiographics. 2010;30:1567-86.

22. Calandriello L, Walsh SLF. Imaging for Sarcoidosis. Semin Respir Crit Care Med. 2017;38:417-36.

23. Abehsera M, Valeyre D, Grenier P, Jaillet H, Battesti JP, Brauner MW. Sarcoidosis with pulmonary fibrosis: CT patterns and correlation with pulmonary function. AJR Am J Roentgenol. 2000;174:1751-7.

24. Scadding JG. Prognosis of intrathoracic sarcoidosis in England. A review of 136 cases after five years' observation. Br Med J. 1961;2:1165-72.

25. Akira M, Kozuka T, Inoue Y, Sakatani M. Long-term follow-up CT scan evaluation in patients with pulmonary sarcoidosis. Chest. 2005;127:185-91.

26. Sobic-Saranovic D, Artiko V, Obradovic V. FDG PET imaging in sarcoidosis. Semin Nucl Med. 2013;43:404-11.

27. Mostard RL, Verschakelen JA, van Kroonenburgh MJ et al. Severity of pulmonary involvement and (18)F-FDG PET activity in sarcoidosis. Respir Med. 2013;107:439-47.

28. Teirstein AS, Machac J, Almeida O, Lu P, Padilla ML, Iannuzzi MC. Results of 188 whole-body fluorodeoxyglucose positron emission tomography scans in 137 patients with sarcoidosis. Chest. 2007;132:1949-53.

29. Zappala CJ, Desai SR, Copley SJ et al. Accuracy of individual variables in the monitoring of long-term change in pulmonary sarcoidosis as judged by serial high-resolution CT scan data. Chest. 2014;145:101-7.

30. Francesqui Candela JR, Llabres M, Brito-Zerón P et al. Pulmonary Function Test and Radiologic Compromise in Sarcoidosis. Available from: https:// www.atsjournals.org/doi/10.1164/ajrccm-conference.2020.201.1_MeetingAbstracts.A1540

31. Rosen Y. Pathology of sarcoidosis. Semin Respir Crit Care Med. 2007;28(1): 36-52.

32. Shigemitsu H, Oblad JM, Sharma OP, Koss MN. Chronic interstitial pneumonitis in end-stage sarcoidosis. Eur Respir J. 2010;35:695-7.

33. Zhang C, Chan KM, Schmidt LA, Myers JL. Histopathology of Explanted Lungs From Patients With a Diagnosis of Pulmonary Sarcoidosis. Chest. 2016;149:499-507.

34. Xu L, Kligerman S, Burke A. End-stage sarcoid lung disease is distinct from usual interstitial pneumonia. Am J Surg Pathol. 2013;37:593-600.

35. Simonneau G, Montani D, Celermajer DS et al. Haemodynamic definitions and updated clinical classification of pulmonary hypertension. Eur Respir J. 2019;53:1801913.

36. Bandyopadhyay D, Humbert M. An update on sarcoidosis-associated pulmonary hypertension. Curr Opin Pulm Med. 2020;26:582-90.

37. Baughman RP, Culver DA, Cordova FC et al. Bosentan for sarcoidosis-associated pulmonary hypertension: a double-blind placebo controlled randomized trial. Chest. 2014;145:810-7.

38. Judson MA, Highland KB, Kwon S et al. Ambrisentan for sarcoidosis associated pulmonary hypertension. Sarcoidosis Vasc Diffuse Lung Dis. 2011;28: 139-45.

39. Milman N, Burton CM, Iversen M, Videbaek R, Jensen CV, Carlsen J. Pulmonary hypertension in end-stage pulmonary sarcoidosis: therapeutic effect of sildenafil? J Heart Lung Transplant. 2008;27:329-34.

40. Nathan SD, Behr J, Collard HR et al. Riociguat for idiopathic interstitial pneumonia-associated pulmonary hypertension (RISE-IIP): a randomised, placebo-controlled phase 2b study. Lancet Respir Med. 2019;7:780-90.

41. Bonham CA, Oldham JM, Gomberg-Maitland M, Vij R. Prostacyclin and oral vasodilator therapy in sarcoidosis-associated pulmonary hypertension: a retrospective case series. Chest. 2015;148:1055-62.

42. Shino MY, Lynch Iii JP, Fishbein MC et al. Sarcoidosis-associated pulmonary hypertension and lung transplantation for sarcoidosis. Semin Respir Crit Care Med. 2014;35:362-71.

43. Pena TA, Soubani AO, Samavati L. Aspergillus lung disease in patients with sarcoidosis: a case series and review of the literature. Lung. 2011;189:167-72.

44. Kantartzis SN, Dacic S, Strollo DC. AIRP best cases in radiologic-pathologic correlation: pulmonary sarcoidosis complicated by aspergilloma formation. Radiographics. 2012;32:469-73.
45. Denning DW, Riniotis K, Dobrashian R, Sambatakou H. Chronic cavitary and fibrosing pulmonary and pleural aspergillosis: case series, proposed nomenclature change, and review. Clin Infect Dis. 2003;37 Suppl 3:S265-80.

46. Fortún J, Meije Y, Fresco G, Moreno S. Aspergilosis. Formas clínicas y tratamiento [Aspergillosis. Clinical forms and treatment]. Enferm Infecc Microbiol Clin. 2012;30:201-8.

47. Patterson TF, Thompson GR 3rd, Denning DW et al. Practice Guidelines for the Diagnosis and Management of Aspergillosis: 2016 Update by the Infectious Diseases Society of America. Clin Infect Dis. 2016;63:e1-e60.

48. Denning DW, Cadranel J, Beigelman-Aubry C et al. Chronic pulmonary aspergillosis: rationale and clinical guidelines for diagnosis and management. Eur Respir J. 2016;47:45-68.

49. Camuset J, Nunes H, Dombret MC et al. Treatment of chronic pulmonary aspergillosis by voriconazole in nonimmunocompromised patients. Chest. 2007;131:1435-41.

50. Setianingrum F, Rautemaa-Richardson R, Shah R, Denning DW. Clinical outcomes of patients with chronic pulmonary aspergillosis managed surgically. Eur J Cardiothorac Surg. 2020;58:997-1003.

51. Farid S, Mohamed S, Devbhandari M et al. Results of surgery for chronic pulmonary Aspergillosis, optimal antifungal therapy and proposed high risk factors for recurrence--a National Centre's experience. J Cardiothorac Surg. 2013;8:180

52. Giron J, Poey C, Fajadet P et al. CT-guided percutaneous treatment of inoperable pulmonary aspergillomas: a study of 40 cases. Eur J Radiol. 1998;28:235-42.

53. Sellarés J, Francesqui J, Llabres M, Hernandez-Gonzalez F, Baughman RP. Current treatment of sarcoidosis. Curr Opin Pulm Med. 2020;26:591-7.

54. Nunes H, Jeny F, Bouvry D, Uzunhan Y, Valeyre D. Indications for treatment of sarcoidosis. Curr Opin Pulm Med. 2019;25:505-18.

55. Rahaghi FF, Baughman RP, Saketkoo LA et al. Delphi consensus recommendations for a treatment algorithm in pulmonary sarcoidosis. Eur Respir Rev. 2020;29:190146.

56. Walsh SL, Wells AU, Sverzellati N et al. An integrated clinicoradiological staging system for pulmonary sarcoidosis: a case-cohort study. Lancet Respir Med. 2014;2:123-30.

57. Mostard RL, Vöö S, van Kroonenburgh MJ et al. Inflammatory activity assessment by F18 FDG-PET/CT in persistent symptomatic sarcoidosis. Respir Med. 2011;105:1917-24.

58. Mostard RL, van Kroonenburgh MJ, Drent M. The role of the PET scan in the management of sarcoidosis. Curr Opin Pulm Med. 2013;19:538-44.

59. Richeldi L, du Bois RM, Raghu G et al. Efficacy and safety of nintedanib in idiopathic pulmonary fibrosis. N Engl J Med. 2014;370:2071-82.

60. Distler O, Highland KB, Gahlemann M et al. Nintedanib for Systemic Sclerosis-Associated Interstitial Lung Disease. N Engl J Med. 2019;380:2518-28.

61. Flaherty KR, Wells AU, Cottin V et al. Nintedanib in Progressive Fibrosing Interstitial Lung Diseases. N Engl J Med. 2019;381:1718-27.

62. Wells AU, Flaherty KR, Brown KK et al. Nintedanib in patients with progressive fibrosing interstitial lung diseases-subgroup analyses by interstitial lung disease diagnosis in the INBUILD trial: a randomised, double-blind, placebo-controlled, parallel-group trial. Lancet Respir Med. 2020;8:453-60.

63. Meyer KC. Lung transplantation for pulmonary sarcoidosis. Sarcoidosis Vasc Diffuse Lung Dis. 2019;36:92-107.

64. Taimeh Z, Hertz MI, Shumway S, Pritzker M. Lung transplantation for pulmonary sarcoidosis. Twenty-five years of experience in the USA. Thorax. 2016;71:378-9.

65. Collins J, Hartman MJ, Warner TF et al. Frequency and CT findings of recurrent disease after lung transplantation. Radiology. 2001;219:503-9.

66. Le Pavec J, Valeyre D, Gazengel P et al. Lung transplantation for sarcoidosis: outcome and prognostic factors [published online ahead of print, 2021 Jan 21]. Eur Respir J. 2021;2003358.

67. Schultz HH, Andersen CB, Steinbruuchel D, Perch M, Carlsen J, Iversen M Recurrence of sarcoid granulomas in lung transplant recipients is common and does not affect overall survival. Sarcoidosis Vasc Diffuse Lung Dis. 2014;31:149-53. 